\title{
Membrane instantons from mirror symmetry
}

\author{
Daniel Robles-Llana, Frank Saueressig, Ulrich Theis \\ AND STEFAN VANDOREN
}

\begin{abstract}
We use mirror symmetry to determine and sum up a class of membrane instanton corrections to the hypermultiplet moduli space metric arising in Calabi-Yau 3-fold compactifications of typeIIA strings. These corrections are mirror to the D1 and $\mathrm{D}(-1)$ brane instantons on the IIB side and are given explicitly in terms of a single function in projective superspace. The corresponding four-dimensional effective action is completely fixed by the Euler number and the genus zero Gopakumar-Vafa invariants of the mirror Calabi-Yau.
\end{abstract}

\section{Introduction}

Over recent years, it has become clear that non-perturbative aspects of string theory play an important role in low-energy effective actions (LEEA) that are relevant for model building. An active area of research has been to determine instanton corrections to superpotentials in $N=1$ flux compactifications, since they can cause moduli stabilization in vacua with a positive cosmological constant. The computation of non-perturbative superpotentials in string or M-theory was initiated in $[1,2]$, following ideas that were developed in [3] for $N=2$ string compactifications. In these approaches, one typically considers the first instanton correction that can be computed using semiclassical methods in string theory. In general, it is very hard to find expressions that are exact to all orders in the string coupling constant $g_{\mathrm{s}}$, unless one can make use of string dualities. For superpotentials in certain $N=1$ flux compactifications this was demonstrated in [4], but for Kähler potentials this is more complicated due to the absence of nonrenormalization theorems (see, however, [5] for some partial results).

In this paper, we study non-perturbative phenomena in string theory in four dimensions with eight supercharges [3] and show that one can find an exact solution for a class of instanton corrections to the LEEA. That is, we are able to determine and sum up an infinite series of membrane instanton corrections to all orders in $g_{\mathrm{s}}$. To obtain these exact results we make use of various dualities and symmetries in $N=2$ compactifications 
of string theories. Moreover, in contrast to the Kähler potential in $N=1$ theories, $N=2$ supersymmetry gives us full control over the perturbative string loop corrections owing to a non-renormalization theorem that prevents corrections beyond one-loop [6].

The models we consider are type-IIA strings compactified on Calabi-Yau 3 -folds (CY) and their mirror versions, type IIB compactified on the mirror $\mathrm{CY}$, without any fluxes turned on. The resulting LEEA contains vector multiplets and hypermultiplets coupled to (ungauged) $N=2$ supergravity. The vector multiplet moduli space is well understood in terms of mirror symmetry, and is not affected by inclusion of D-branes. Mirror symmetry between the hypermultiplet moduli spaces in IIA and IIB is complicated by the fact that both moduli spaces receive stringy perturbative and non-perturbative corrections. The perturbative corrections are one-loop (as higher order corrections can be absorbed in field redefinitions in the LEEA) and are proportional to the Euler number, consistent with mirror symmetry [6]. The non-perturbative corrections are much more complicated, and their full form is unknown at present. However, their generic structure was argued in [3] to be as follows. On the type IIB side non-perturbative corrections are given by instantons arising from odd-dimensional Euclidean D-branes wrapping even supersymmetric cycles, together with NS5/D5-branes wrapping the entire CY. In type IIA they are given by membranes wrapping supersymmetric three-cyles and NS5-branes again wrapping the entire CY. We will consider membrane instantons only, i.e., Euclidean D2-branes wrapping supersymmetric three-cycles in the CY, leaving the NS5-brane instantons for future research. We comment on how to determine these corrections in figure 1 . More precisely, we consider membrane instantons coming from D2-branes wrapping only half of the three-cycles, say the "electric" or $A$-cycles. These are related to our recent results on $\mathrm{D} 1$ and $\mathrm{D}(-1)$ instanton corrections on the type IIB side [7] by mirror symmetry in the presence of D-branes [8]. Geometrically, under the mirror map holomorphic two-cycles in the CY are exchanged by special Lagrangian three-cycles in the mirror CY.

The main result of this paper is the implementation of the mirror map and the explicit form of the instanton corrected hypermultiplet geometry. In fact, recently the instanton corrections were determined in [9] in the conifold limit of type IIB strings upon shrinking the size of a two-cycle. The results showed precise agreement with the membrane instantons obtained in [10] in type-IIA strings on the mirror $\mathrm{CY}$, thereby providing a non-perturbative test of mirror symmetry in the conifold limit. Here, we assume mirror symmetry to hold at a generic point in the moduli space, and use it to determine the instanton corrections to the effective action away from the conifold 


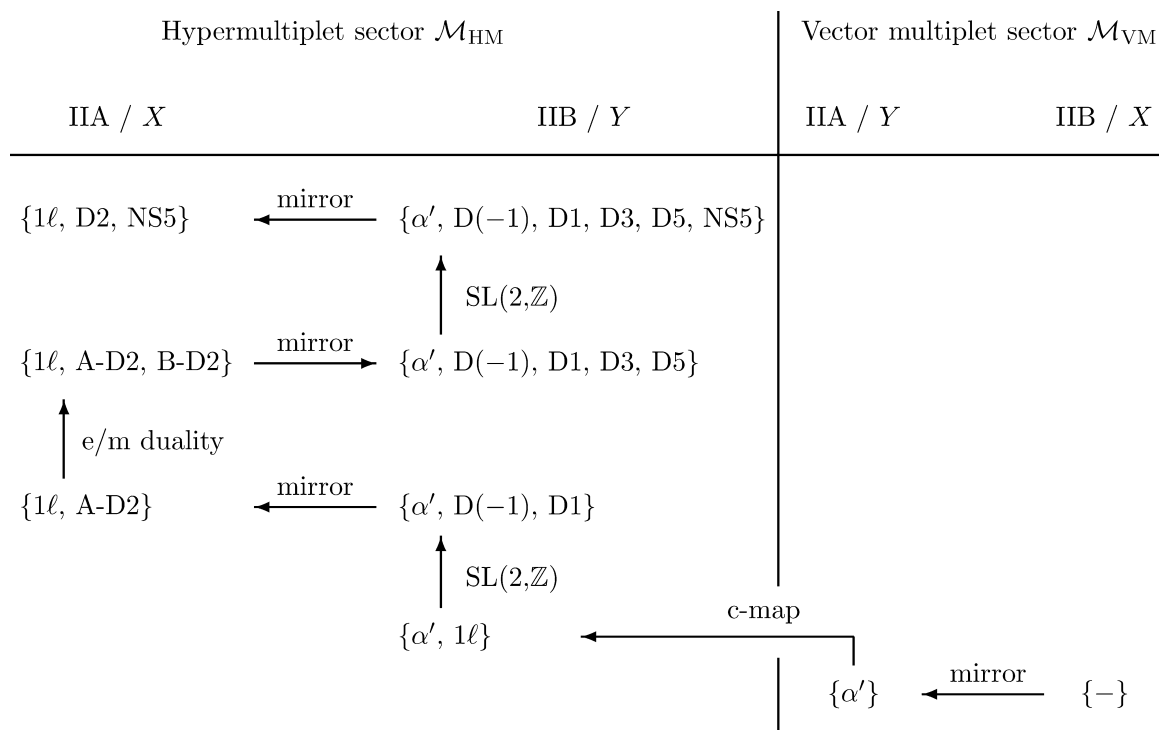

Figure 1: Prospective duality chain for determining the full quantum LEEA of type II strings compactified on a generic CY $X$, and its mirror partner $Y$. In the vector multiplet sector there are $\alpha^{\prime}$ corrections that appear on the IIA side only and can be obtained via mirror symmetry; they comprise worldsheet loop and instanton corrections. The c-map transfers these into the IIB hypermultiplet sector. In addition, there is a one-loop $g_{\mathrm{s}}$ correction, in the figure denoted by $1 \ell$, determined in $[6]$. Imposing $\operatorname{SL}(2, \mathbb{Z})$ invariance produces the non-perturbative corrections arising from D1-brane and more general $(p, q)$-string instantons as well as $\mathrm{D}(-1)$ instantons [7]. The latter naturally combine with the perturbative $\alpha^{\prime}$ and $g_{\mathrm{s}}$ corrections. As shown in this paper, applying mirror symmetry to these corrections gives rise to the $A$-cycle D2-brane instanton contributions on the IIA side. Though beyond the scope of this paper, one may now continue to employ various dualities that should in principle produce all possible quantum corrections: using electromagnetic $(\mathrm{e} / \mathrm{m})$ duality to impose symplectic invariance will give the $B$-cycle D2-brane instantons. Mirror symmetry will map these to the as of yet unknown D3- and D5-brane instanton corrections on the IIB side. Another application of $\mathrm{SL}(2, \mathbb{Z})$ duality then will give rise to pure NS5-brane and D5-NS5 bound state instantons. Finally, applying mirror symmetry one last time will produce the NS5-brane corrections on the IIA side. 
limit, where the hypermultiplet moduli space is quaternion-Kähler instead of hyperkähler.

Finding the non-perturbative corrections to the hypermultiplet moduli space by means of a microscopic instanton calculation has been a notoriously complicated problem. However, for rigid CY manifolds $\left(h_{1,2}=0\right)$, yielding only the universal hypermultiplet, a microscopic string theory calculation was performed in [11] following the procedure outlined in [3]. In this approach, the one-instanton sector can be determined in the semiclassical approximation, up to an overall numerical coefficient that comes out of the one-loop determinant of fluctuations around the instanton. The method used in the present paper fixes this coefficient in terms of the Euler number of the CY and allows us to go beyond the semiclassical approximation.

Finally, we wish to point out that if the CY is $K 3$-fibered, our results are related to a class of worldsheet instantons in the heterotic string on $K 3 \times T^{2}$ using string duality. The mapping of membrane instantons to worldsheet instantons was recently investigated in [12], and it would be interesting to study further how our results fit into that framework.

In the next section, we briefly review some general aspects of off-shell effective actions for hypermultiplet and tensor multiplet couplings to $N=2$ supergravity that are needed in later sections. In Section 3, we present the connection with the string theory variables for both the type-IIA and -IIB theories and discuss the breaking of isometries by instantons. Section 4 deals with microscopic aspects of mirror symmetry, including both the NS-NS and RR sectors, and we construct the mirror map between the IIA and IIB hypermultiplet moduli spaces. Finally, in Section 5, we implement mirror symmetry to determine the membrane instanton correction starting from previously known results on D1 and D $(-1)$ instantons in IIB. We close with some conclusions and further discussions on Section 6. Two appendices are included at the end with technical details and useful formulas.

\section{Off-shell effective actions}

In this section, we describe general aspects of superspace LEEA for hypermultiplets and their dual tensor multiplets. After a short review of projective superspace, we give the resulting tree-level and one-loop effective actions for type II strings compactified on a CY.

The total moduli space $\mathcal{M}$ for a type II string compactification on a generic CY locally factorizes into a direct product $\mathcal{M}=\mathcal{M}_{\mathrm{VM}} \times \mathcal{M}_{\mathrm{HM}}$, where $\mathcal{M}_{\mathrm{VM}}$ and $\mathcal{M}_{\mathrm{HM}}$ denote the vector and hypermultiplet moduli space. 


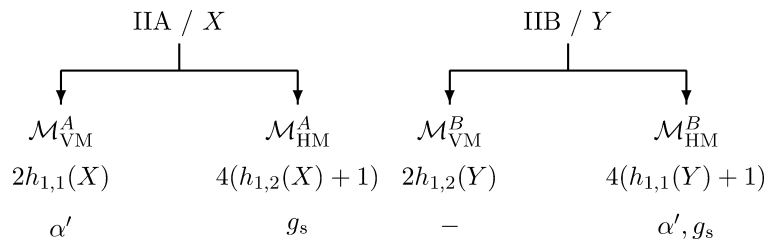

Figure 2: Massless matter spectrum arising in CY compactifications of type II strings. The moduli spaces for vector and hypermultiplets are denoted by $\mathcal{M}_{\mathrm{VM}}$ and $\mathcal{M}_{\mathrm{HM}}$, respectively, with their real dimensions below in terms of the Hodge numbers $h_{p, q}$ of the CY. The last line indicates possible quantum corrections to the respective sector. Here $\alpha^{\prime}$ and $g_{\mathrm{s}}$ denote corrections from the worldsheet conformal field theory and in the (four-dimensional) string coupling constant, respectively. Note that the vector multiplet sector of the type IIB compactification is classically exact.

The metrics on $\mathcal{M}_{\mathrm{VM}}^{\mathrm{IIA}} / X$ and $\mathcal{M}_{\mathrm{VM}}^{\mathrm{IIB}} / Y$ are entirely determined by the prepotentials $F(X)$ on the moduli spaces of (complexified) Kähler deformations on $X$ and complex structure deformations on $Y$, respectively. As the fourdimensional dilaton belongs to a hypermultiplet, the generic factorization of the moduli space implies that the vector moduli spaces are exact at string tree-level. This is summarized in figure 2 .

The scalars of the hypermultiplets coupled to $N=2$ supergravity parametrize a quaternion-Kähler manifold [13]. For type-IIA (type-IIB) compactifications, this manifold is spanned by $h_{1,2}+1\left(h_{1,1}+1\right)$ physical hypermultiplets. Generic hypermultiplet couplings do not admit an off-shell description with a finite number of auxiliary fields, so no simple superspace formula exists for the effective action. As we explain in the next section, for the problem at hand, there will be a suitable number of Peccei-Quinn-like isometries that act as shifts on some of the scalars. In this case, the hypermultiplets have a dual description in terms of $N=2$ tensor multiplets which allow for an off-shell description with a finite number of auxiliary fields in projective superspace $[14,15]$. The $N=2$ tensor multiplets are written as

$$
\eta^{I}(\zeta)=\frac{v^{I}}{\zeta}+x^{I}-\bar{v}^{I} \zeta
$$

where $v$ and $x$ denote $N=1$ chiral and real linear (containing a real scalar and a tensor) superfields, respectively, while $\zeta$ is a complex coordinate on the Riemann sphere. In rigid $N=2$ supersymmetry, superspace Lagrangian 
densities for the tensor multiplets can be written as

$$
\mathcal{L}\left(x^{I}, v^{I}, \bar{v}^{I}\right)=\operatorname{Im} \oint_{\mathcal{C}} \frac{d \zeta}{2 \pi i \zeta} H\left(\eta^{I}(\zeta), \zeta\right)
$$

with $\mathcal{C}$ an appropriately chosen contour that typically encloses the poles and branch cuts of the function $H$. Supersymmetry does not pose any constraints on $\mathcal{C}$. By construction $\mathcal{L}$, when taken as a function of the scalar fields, automatically satisfies the constraint for rigid supersymmetry

$$
\mathcal{L}_{x^{I} x^{J}}+\mathcal{L}_{v^{I} \bar{v}^{J}}=0
$$

where $\mathcal{L}_{x^{I}}$ denotes the derivative of $\mathcal{L}$ with respect to $x^{I}$, etc.

The tensor multiplet action is obtained by integrating the superspace Lagrangian $\mathcal{L}$ over half of superspace, with coordinates $\theta_{i \alpha}, \bar{\theta}_{\dot{\alpha}}^{i}$, where $i=1,2$ is an $\mathrm{SU}(2)_{R}$ index and $\alpha, \dot{\beta}=1,2$ are Lorentz spinor indices. For vector multiplet actions, one integrates superspace densities over a chiral subspace spanned by the $\theta_{i \alpha}$ only. Such terms are called F-terms. For tensor multiplets, one chooses a different subspace spanned by, say, $\theta_{1 \alpha}$ and $\bar{\theta}_{\dot{\alpha}}^{2}$. Such terms can be called twisted F-terms, and the action for the tensor multiplets then takes the form

$$
S=\int d^{4} x d^{2} \theta d^{2} \bar{\theta} \mathcal{L}
$$

For heterotic superstrings on $K 3 \times T^{2}$, which are dual to our models, projective superspace arises naturally from the hybrid formalism [16], whereas for type II strings on a CY the situation is less clear; see [17] for more details. Superspace effective actions have also been studied in [18].

To couple the resulting tensor multiplet action to supergravity, we use the superconformal calculus and introduce a compensating tensor multiplet. The theory can then be made scale invariant, and the constraints from conformal symmetry lead to the conditions that $H$ has no explicit $\zeta$ dependence and is homogeneous of degree 1 under the contour integral [19]. The resulting expression is then conformally coupled to the Weyl multiplet, which contains the degrees of freedom of $N=2$ conformal supergravity; it is the gauge multiplet of the $N=2$ superconformal algebra. Gauge-fixing the $\mathrm{SU}(2)_{R}$ and dilatations eventually gives the tensor multiplet couplings to Poincaré supergravity in component language [20]. What is important here is that the entire supergravity action is determined by a single function $\mathcal{L}(x, v, \bar{v})$ of the tensor multiplet scalar components. 
At the superconformal level the dualization of the tensor multiplets to hypermultiplets can be done by performing a Legendre transform on the superspace Lagrangian density $\mathcal{L}$ [21]. The resulting function is the hyperkähler potential on the hyperkähler cone describing superconformally coupled hypermultiplets [22]. Taking the superconformal quotient as in [19] leads to the quaternion-Kähler hypermultiplet moduli space.

Before doing the Legendre transform, one can construct a tensor potential [20]

$$
\chi\left(x^{I}, v^{I}, \bar{v}^{I}\right)=x^{I} \mathcal{L}_{x^{I}}-\mathcal{L},
$$

which satisfies the differential relations

$$
\mathcal{L}_{x^{I} x^{J}}=\frac{1}{2}\left(\chi_{x^{I} x^{J}}+\chi_{v^{I} \bar{v}^{J}}\right) .
$$

The two functions $\chi$ and $\mathcal{L}$ will play the central role in our discussion; they encode all the quantum corrections, from string loops as well as from instantons. It turns out to be particularly convenient to work with $\chi$, since symmetries of the effective action directly translate to invariances of the tensor potential. In Section 5, we will give explicit formulas for the instanton corrections to both of these functions. Further details will also be given in Appendix B.

At string tree-level, the hypermultiplet moduli space can be determined from the vector multiplet effective action in the T-dual theory by applying the c-map [23]. The resulting quaternion-Kähler geometry was worked out in [24], and the corresponding superspace Lagrangian takes the simple form $[25,26]$ (also see [18])

$$
\mathcal{L}=\operatorname{Im} \oint_{\mathcal{C}_{0}} \frac{d \zeta}{2 \pi \mathrm{i} \zeta} \frac{F\left(\eta^{\Lambda}\right)}{\eta^{0}}
$$

Here, $F$ is the vector multiplet prepotential of the T-dual theory, homogeneous of degree 2 , but with the vector multiplets $X^{\Lambda}$ replaced by tensor multiplets $\eta^{\Lambda}$. (See table 1 for index ranges.) Finally, $\eta^{0}$ can be chosen to be the conformal compensator. Equation (2.7) was first derived in a certain $\mathrm{SU}(2)_{R}$ gauge $\left(v^{0}=0\right)$ with the contour $\mathcal{C}_{0}$ chosen around the origin. It was then rederived in [27] in a gauge-independent way with the contour $\mathcal{C}_{0}$ around one of the zeroes, say $\zeta_{+}$, of $\zeta \eta^{0}$. In the gauge $v^{0}=0$ one recovers the results of [25]. 
Table 1: Summary of indices used in the hypermultiplet sector. The index $a$ runs over one value less than $\Lambda$, making the coordinates $z^{a}$ projective, and $I=\{0, \Lambda\}$ also includes the compensator. For $X$ and $Y$ mirror to each other, $h_{1,2}(X)=h_{1,1}(Y)$ so that the number of multiplets agrees.

\begin{tabular}{ll}
\hline type-IIA strings on $X$ & type-IIB strings on $Y$ \\
\hline$I=0,1, \ldots, h_{1,2}(X)+1$ & $I=0,1, \ldots, h_{1,1}(Y)+1$ \\
$\Lambda=1, \ldots, h_{1,2}(X)+1$ & $\Lambda=1, \ldots, h_{1,1}(Y)+1$ \\
$a=2, \ldots, h_{1,2}(X)+1$ & $a=2, \ldots, h_{1,1}(Y)+1$ \\
\hline
\end{tabular}

At one-loop order in $g_{\mathrm{s}}$, the resulting action is given by $[6]^{1}$

$$
\mathcal{L}= \pm \operatorname{Im} \frac{\mathrm{i} \chi_{E}}{24 \pi} \oint_{\mathcal{C}_{1}} \frac{d \zeta}{2 \pi \mathrm{i} \zeta} \eta^{0} \ln \eta^{0}
$$

with the positive sign for IIB and the negative sign for type IIA. Here, $\chi_{E}$ is the Euler number of the CY. Notice that the results for type IIA and IIB are consistent with mirror symmetry, since the Euler number switches sign for the mirror $\mathrm{CY}$. The contour $\mathcal{C}_{1}$ is taken around the logarithmic branch cut between the origin and $\zeta_{+}[28]$. The formula (2.8) generalizes the results for the universal hypermultiplet obtained in [29,30]. Moreover, in [29] it was shown that higher loop corrections can be absorbed by field redefinitions. The effective absence of higher-loop corrections was given an explanation in [6] in terms of a non-renormalization theorem in projective superspace, where it can be generalized to an arbitrary number of hypermultiplets.

\section{String theory variables}

In the previous section, we gave generic formulas for tensor-/hypermultiplet LEEA in string perturbation theory. To connect these formulas to a specific string theory compactification, we need to specify the relation between the tensor multiplet components and the four-dimensional physical fields arising from the dimensional reduction of the ten-dimensional massless modes,

\footnotetext{
${ }^{1}$ We use a slightly different normalization for the prepotential than in [6]. As a result, the overall numerical coefficient in (2.8) relative to the classical term (2.7) is different. With the conventions used in this paper, the monodromies of the prepotential around the conifold point are integer-valued, as is common in the literature.
} 
which are given by conformally and $\mathrm{SU}(2)_{R}$ invariant combinations of the tensor multiplet scalars. In this section, we state these relations for type-IIB and type-IIA compactifications, and we analyze the breaking of isometries due to instantons. In view of the discussion of mirror symmetry in the next section, it is thereby useful to distinguish between the NS-NS and RR sectors of the compactification.

\subsection{Type IIB}

In type IIB compactifications on a CY manifold $Y$, the four-dimensional fields organize themselves into the gravitational multiplet, $h_{1,2}(Y)$ vector multiplets, $h_{1,1}(Y)$ tensor multiplets, and a double-tensor multiplet [31]. The latter contains two tensors and two scalars and has an off-shell description in terms of two tensor multiplets, which contain the four compensating scalar fields that restore the dilatation and $\mathrm{SU}(2)_{R}$ symmetries. Thus, offshell there are $h_{1,1}(Y)+2$ tensor multiplets.

The NS-NS sector of the compactification contains the ten-dimensional dilaton $\phi$ and the complexified Kähler moduli $z^{a}$ arising from integrating the Kähler form $J$ and the NS two-form $B_{\mathrm{NS}}$ over a basis of two-cycles $\gamma_{(2)}^{a}$ of $H_{2}(Y, \mathbb{Z})$

$$
z^{a}=b^{a}+\mathrm{i} t^{a}=\int_{\gamma_{(2)}^{a}}\left(B_{\mathrm{NS}}+\mathrm{i} J\right), \quad a=2, \ldots, h_{1,1}+1 .
$$

The dilaton provides one of the scalars of the double tensor multiplet, while the $z^{a}$ account for $2 h_{1,1}(Y)$ of the tensor multiplet scalars.

In the RR sector, the axion coming from the zero form $C_{0}$ corresponds to the second scalar in the double-tensor multiplet, while the periods of the RR two-form $C_{2}$,

$$
c^{a}=\int_{\gamma_{(2)}^{a}} C_{2}
$$

make up the remaining $h_{1,1}(Y)$ scalars. Furthermore, the four-dimensional tensor fields are provided by the space-time parts of $B_{\mathrm{NS}}$ and $C_{2}$ together with $h_{1,1}(Y)$ tensors arising as the periods of the $\mathrm{RR}$ four-form $C_{4}$ with respect to $\gamma_{(2)}^{a}$. The dilaton-axion system can be combined into the complex scalar

$$
\tau=\tau_{1}+\mathrm{i} \tau_{2}=C_{0}+\mathrm{i} e^{-\phi}
$$


The relation between the "microscopic" scalars $\tau, z^{a}, c^{a}$ and the scalars appearing in the tensor multiplets $(2.7)$ is given by [27]

$$
\begin{aligned}
\tau & =\frac{1}{\left(r^{0}\right)^{2}}\left(\vec{r}^{0} \cdot \vec{r}^{1}+\mathrm{i}\left|\vec{r}^{0} \times \vec{r}^{1}\right|\right), \\
z^{a} & =\frac{\eta_{+}^{a}}{\eta_{+}^{1}}, \quad c^{a}=\frac{\left(\vec{r}^{0} \times \vec{r}^{1}\right) \cdot\left(\vec{r}^{1} \times \vec{r}^{a}\right)}{\left|\vec{r}^{0} \times \vec{r}^{1}\right|^{2}} .
\end{aligned}
$$

Here we have introduced

$$
\vec{r}^{I}=\left[2 v^{I}, 2 \bar{v}^{I}, x^{I}\right], \quad \vec{r}^{I} \cdot \vec{r}^{J}=2 v^{I} \bar{v}^{J}+2 v^{J} \bar{v}^{I}+x^{I} x^{J}
$$

and $\eta_{+}^{\Lambda}=\eta^{\Lambda}\left(\zeta_{+}\right)$, where $\zeta_{+}$is one of the roots of $\zeta \eta^{0}(\zeta)$. See Appendix B for more details.

In the large volume limit, $b^{a}$ and $c^{a}$ are subject to continuous PecceiQuinn shift symmetries. Non-perturbatively, these symmetries are broken to discrete subgroups by worldsheet instantons and D1 instantons, respectively. Similarly, the shift symmetry of the axion $\tau_{1}$ is broken by $\mathrm{D}(-1)$ instantons. In our set-up, we are still left with $h_{1,1}+2$ tensors. Dualizing these tensors to scalars again leads to $h_{1,1}+2$ continuous shift symmetries. These will also be broken by instantons; the first $h_{1,1}$ by D3-brane instantons, and the two remaining ones, originating from the double-tensor multiplet, by D5 and NS5-brane instantons. Including these instanton corrections require a formulation in terms of hypermultiplets which are not dual to tensor multiplets, so the tensor multiplet framework used in this paper is no longer applicable.

\subsection{Type IIA}

When compactifying type-IIA strings on $X$, the off-shell formulation of the hypermultiplet sector uses $h_{1,2}(X)+2$ tensor multiplets. Not counting the conformal compensators, consisting of four scalars, we therefore have $3 h_{1,2}+$ 2 physical scalars and $h_{1,2}+2$ tensors.

The NS-NS sector contains the dilaton $\phi$ and the complex structure deformations of $X$, which together account for $2 h_{1,2}(X)+1$ scalars. In order to find the coordinates on the complex structure moduli space, we introduce a symplectic basis of $h_{1,2}(X)+1 A$ - and $B$-cycles $\left\{\gamma_{(3)}^{\Lambda}, \gamma_{\Lambda}^{(3)}\right\}$ of $H_{3}(X, \mathbb{Z})$. The complex structure deformations can then be parametrized by the periods of the holomorphic three-form $\Omega$ with respect to the $A$-cycles $\gamma_{(3)}^{\Lambda}$. Choosing $\gamma_{(3)}^{1}$ to be the cycle dual to $\Omega$, the inhomogeneous (physical) 
complex structure moduli $z^{a}$ are defined as

$$
X^{\Lambda}=\int_{\gamma_{(3)}^{\Lambda}} \Omega, \quad z^{\Lambda} \equiv \frac{X^{\Lambda}}{X^{1}}=\left(1, z^{a}\right)
$$

In the $\mathrm{RR}$ sector, the periods of the $\mathrm{RR}$ three-form $C_{3}$

$$
A^{\Lambda}=\int_{\gamma_{(3)}^{\Lambda}} C_{3}
$$

give the remaining $h_{1,2}(X)+1$ physical scalars. In principle, integrating $C_{3}$ over the dual $B$-cycles gives rise to $h_{1,2}(X)+1$ additional scalars $B_{\Lambda}$. In our framework, these are dualized into tensors. Together with the space-time part of $B_{\mathrm{NS}}$ this gives $h_{1,2}+2$ tensors, so the total number of degrees of freedom works out correctly. Notice that upon interchanging $h_{1,1}$ with $h_{1,2}$, the IIA spectrum precisely agrees with the one for the type IIB theory. This is of course a manifestation of mirror symmetry. At the classical level, the mirror map relating the tree-level IIA and IIB hypermultiplet moduli spaces was found to be [31]

$$
\phi_{\mathrm{IIA}}=\phi_{\mathrm{IIB}}, \quad A^{1}=\tau_{1}, \quad A^{a}=-\left(c^{a}-\tau_{1} b^{a}\right), \quad z_{\mathrm{IIA}}^{a}=z_{\mathrm{IIB}}^{a}
$$

At the classical level, the RR fields $A^{\Lambda}$ have continuous Peccei-Quinn isometries which will be broken to a discrete subgroup by the instanton corrections. Using (3.4), the type-IIA scalars $e^{-\phi}, z^{a}, A^{\Lambda}$ are related to the tensor multiplet components via

$$
e^{-\phi}=\frac{1}{\left(r^{0}\right)^{2}}\left|\vec{r}^{0} \times \vec{r}^{1}\right|, \quad z^{a}=\frac{\eta_{+}^{a}}{\eta_{+}^{1}}, \quad A^{\Lambda}=\frac{1}{\left(r^{0}\right)^{2}}\left(\vec{r}^{0} \cdot \vec{r}^{\Lambda}\right) .
$$

In the next section, we show that the mirror map (3.8), in fact, also holds in the presence of instantons, but with additional periodic identifications of the fields.

Dualizing the scalars $B_{\Lambda}$ into tensor fields and keeping the NS two-form makes the associated shift symmetries manifest. Non-perturbatively these symmetries are broken by membrane instantons that wrap the $B$-cycles and by NS5-brane instantons, respectively. These instanton corrections are not taken into account in our analysis, since their inclusion cannot be described by our off-shell tensor multiplet description. 


\section{Mirror symmetry}

Mirror symmetry can be phrased as the statement that the A-model topological amplitudes on a CY $X$ can be mapped to the B-model topological amplitudes of the mirror CY $Y$. At the level of the four-dimensional LEEA this implies that the vector multiplet moduli spaces $\mathcal{M}_{\mathrm{VM}}^{\mathrm{IIA}} / Y$ and $\mathcal{M}_{\mathrm{VM}}^{\mathrm{IIB}} / X$ are isomorphic, i.e., there is a choice of holomorphic coordinates for which the prepotentials (computed from the genus zero topological amplitude) underlying these spaces agree. Via the c-map (2.7) this entails that the hypermultiplet moduli spaces $\mathcal{M}_{\mathrm{HM}}^{\mathrm{IIA}} / X$ and $\mathcal{M}_{\mathrm{HM}}^{\mathrm{IIB}} / Y$ agree at string tree-level. This version of mirror symmetry is by now well established, see, e.g., [32].

For the purpose of this paper in which we also include D-branes, we need a version of mirror symmetry that includes D-branes, in particular wrapped Euclidean D-branes representing spacetime instantons. At the level of the LEEA of type-IIA/IIB string theory, this version of mirror symmetry states that $\mathcal{M}_{\mathrm{HM}}^{\mathrm{IIA}} / X$ and $\mathcal{M}_{\mathrm{HM}}^{\mathrm{IIB}} / Y$ are also isomorphic once non-perturbative corrections are included. In this section, we assume mirror symmetry to hold and determine the mirror map to relate the IIB and IIA hypermultiplet moduli spaces non-perturbatively.

\subsection{Mirror symmetry at string tree-level}

Mirror symmetry between the prepotentials of complexified Kähler/complex structure deformations of mirror CY's is naturally formulated in terms of two specific kinds of coordinates. On the complexified Kähler moduli space of a CY Y, the natural coordinates arise through the expansion around the large volume limit of $Y$. From the perspective of the $N=2$ non-linear sigma model with target space $Y$, they appear in the exponentials of the threepoint correlation functions of chiral primary operators $\mathcal{O}_{a}$ associated with classes $A_{a}$ in $H^{1,1}(Y, \mathbb{C})$ as computed from the topological A-model

$$
\left\langle\mathcal{O}_{a} \mathcal{O}_{b} \mathcal{O}_{c}\right\rangle=\int_{Y} A_{a} \wedge A_{b} \wedge A_{c}+\sum_{k_{d}} N_{a b c}^{k_{d}} e^{2 \pi i k_{d} z^{d}} .
$$

Here $k_{a}$ runs over homology classes of rational curves in $Y$ and $N_{a b c}^{k_{d}}$ are integers given by the intersection theory on the moduli space of rational curves, which can be related to the genus zero Gopakumar-Vafa invariants of $Y$. The expression (4.1) is the third derivative of the prepotential, which can then be found by integration. The coordinates $z^{a}$ agree with the coordinates provided by the IIB moduli fields (3.1). 
The canonical coordinates on the moduli space of complex structures of the mirror CY $X$ are the so-called "flat" coordinates arising from an expansion of the prepotential around the large complex structure limit of $X$. The coordinates mirror to the $z_{\mathrm{IIB}}^{a}$ appearing in the large volume expansion of the prepotential (4.1) can be constructed as follows [33,34]. Using the so-called monodromy weight filtration [35], one chooses a symplectic basis $\left\{\gamma_{(3)}^{\Lambda}, \gamma_{\Lambda}^{(3)}\right\}$ of $H_{3}(X, \mathbb{Z})$ such that the cycle $\gamma_{(3)}^{1}$ spans $H_{0,3}(X, \mathbb{Z})$ (the dual of the class $H^{3,0}(X)$ given by $\left.\Omega(X)\right)$. It is singled out by having trivial monodromy at the large complex structure limit. The other cycles $\gamma_{(3)}^{a}$ constitute a basis of $H_{1,2}(X, \mathbb{Z})$ and can be shifted in integer multiples of $\gamma_{(3)}^{1}$ by monodromy transformations of $H_{3}(X, \mathbb{Z})$ around the large complex structure limit. ${ }^{2}$ The flat (inhomogeneous) coordinates on the moduli space of complex structures are then the normalized periods of $\Omega$ introduced in (3.6). The period integrals over the dual $B$-cycles $\gamma_{\Lambda}^{(3)}$ define first derivatives of the prepotential on the complex structure moduli space of $X$

$$
F_{\Lambda}(X)=\int_{\gamma_{\Lambda}^{(3)}} \Omega
$$

The prepotential is obtained by using homogeneity, $X^{\Lambda} F_{\Lambda}=2 F(X)$.

In terms of the specific coordinates (3.1) and (3.6), mirror symmetry between the CY's $Y$ and $X$ is the statement that, under the identification (called the mirror map [34])

$$
z_{\mathrm{IIB}}^{a} \equiv \int_{\gamma_{(2)}^{a}}\left(B_{\mathrm{NS}}+i J\right)=\frac{\int_{\gamma_{(3)}^{a}} \Omega}{\int_{\gamma_{(3)}^{1}} \Omega} \equiv z_{\mathrm{IIA}}^{a},
$$

the prepotentials derived from (4.1) and (4.2) agree. The exponential worldsheet instanton corrections to the former arise from the expansion of the dual period integrals around the large complex structure limit.

Furthermore, agreement between the hypermultiplet moduli spaces requires the identification of the type-IIA and -IIB dilatons:

$$
\phi_{\mathrm{IIA}}=\phi_{\mathrm{IIB}}
$$

Since mirror symmetry is supposed to work order by order in string perturbation theory, we will assume this identification to hold throughout. To simplify our notation we will denote $\tau_{2}=e^{-\phi_{\text {IIA }}}=e^{-\phi_{\text {IIB }}}$ resorting to (4.4) implicitly.

\footnotetext{
${ }^{2}$ See [35] for more mathematical details.
} 


\subsection{Inclusion of D-branes}

Quantum mirror symmetry between type IIA on $X$ and type IIB on $Y$ also requires matching the non-perturbative string corrections in the respective hypermultiplet sectors. The corrections of interest in this paper are the ones found in [7] and come from $\mathrm{D}(-1)$ and D1-brane instantons in type IIB on $Y$. In this subsection, we discuss generic aspects of the mirror symmetry between these objects and D2-branes in type IIA on $X$ and review the mirror map between the relevant supersymmetric cycles and RR fields, which we implement at the level of the LEEA in the next section.

Let us start by considering the D-brane worldvolume actions in type IIB and type IIA, respectively. The $\mathrm{D}(-1)$ instanton couples to the RR scalar $C_{0}=\tau_{1}$, and its action is given by $^{3}$

$$
S_{\mathrm{D}(-1)}=2 \pi \tau_{2}+2 \pi i \tau_{1}
$$

For D1-brane instantons wrapping a two-cycle with homology $k_{a} \gamma_{(2)}^{a}$, supersymmetry imposes that the map embedding the worldvolume $\Sigma$ into the two-cycle be holomorphic and that the field strength $F=d A$ of the worldvolume gauge field $A$ is such that $F / 2 \pi$ is an integral harmonic form on any holomorphic two-cycle $[3,36]$. The worldvolume action $S_{\text {DBI }}+S_{\text {Top }}$ for such a D1-instanton is given by [36]

$$
\begin{aligned}
S_{\mathrm{D} 1 / k_{a} \gamma_{(2)}^{a}=} & 2 \pi \int_{\Sigma} \tau_{2} \sqrt{\operatorname{det}\left(\hat{G}+\left(F / 2 \pi-\hat{B}_{\mathrm{NS}}\right)\right)} \\
& +2 \pi i \int_{\Sigma} e^{F / 2 \pi-\hat{B}_{\mathrm{NS}}} \wedge\left(\hat{C}_{0}+\hat{C}_{2}\right) \\
= & 2 \pi \tau_{2} \sqrt{\left(k_{a} t^{a}\right)^{2}+\left(k_{a} b^{a}+n\right)^{2}}+2 \pi i\left(k_{a} c^{a}-\tau_{1}\left(k_{a} b^{a}+n\right)\right) .
\end{aligned}
$$

In the above formula, $\hat{G}$ and $\hat{B}$ denote the pullback of the space-time fields $G$ and $B$, and $n$ is an arbitrary integer which encodes the flux of $F$ on the two-cycle (i.e., the first Chern class of the U(1) bundle). Because of the way the integer $n$ appears in the topological Chern-Simons coupling in (4.6), it is naturally associated with $\mathrm{D}(-1)$ charge inside the D1-instanton.

We now turn to the type-IIA compactification on $X$. Recall that in the large volume limit, mirror symmetry aligns the integer homologies $\bigoplus H_{2 k}$ $(Y, \mathbb{Z})$ and $H_{3}(X, \mathbb{Z})$. More precisely, the zero homology $H_{0}(Y, \mathbb{Z})$ is mapped to $H_{0,3}(X, \mathbb{Z})[37]$. In the basis $(3.6)$, the homology $H_{0,3}(X, \mathbb{Z})$ is spanned

\footnotetext{
${ }^{3}$ Here and henceforth we are setting $4 \pi^{2} \alpha^{\prime}=1$. Furthermore, the inverse string coupling constant is given by $g_{s}^{-1}=\tau_{2}$.
} 
by the three-cycle $\gamma_{(3)}^{1}$. As a consequence, a $\mathrm{D}(-1)$-instanton wrapping a zero-cycle in $Y$ maps to a D2-instanton wrapping the three-cycle $\gamma_{(3)}^{1}$ in $X$ [8]. The SYZ construction of mirror symmetry [38] then allows to identify $\gamma_{(3)}^{1}$ as a special Lagrangian cycle with $T^{3}$ topology. This implies that also the moduli spaces of the $\mathrm{D}(-1)$ and $\mathrm{D} 2 / \gamma_{(3)}^{1}$ instantons agree.

For the two-cycles mirror symmetry aligns $H_{1,1}(Y, \mathbb{Z})$ with $H_{1,2}(X, \mathbb{Z})$ and takes integral bases $\gamma_{(2)}^{a}$ to $\gamma_{(3)}^{a}$ after a suitable linear transformation. As a consequence of the mixing of zero-forms and two-forms in the coupling of the D1-branes to $\left(F / 2 \pi-\hat{B}_{\mathrm{NS}}\right)$ in $(4.6)$, the mirror of a D1-instanton wrapping the holomorphic two-cycle in homology $k_{a} \gamma_{(2)}^{a}$ with $n$ units of flux and action (4.6) is given by a D2-instanton wrapping a special Lagrangian cycle in class $k_{a} \gamma_{(3)}^{a}+n \gamma_{(3)}^{1}$ [8]. Equivalently, a shift of the $B$-field $k_{a} b^{a} \mapsto k_{a} b^{a}+n$ is mirror to a monodromy transformation $k_{a} \gamma_{(3)}^{a} \mapsto k_{a} \gamma_{(3)}^{a}+n \gamma_{(3)}^{1}[35]$.

Based on these considerations the worldvolume action of the D2-brane instanton mirror to (4.5) is given by

$$
S_{\mathrm{D} 2 / \gamma_{(3)}^{1}}=2 \pi \tau_{2}+2 \pi i A^{1},
$$

while the worldvolume action of the D2-brane instanton mirror to (4.6) is

$$
\begin{aligned}
S_{\mathrm{D} 2 / k_{a} \gamma_{(3)}^{a}+n \gamma_{(3)}^{1}} & =2 \pi \tau_{2}\left|\int_{k_{a} \gamma_{(3)}^{a}+n \gamma_{(3)}^{1}} \Omega\right|-2 \pi i \int_{k_{a} \gamma_{(3)}^{a}+n \gamma_{(3)}^{1}} \hat{C}_{3} \\
& =2 \pi \tau_{2}\left|n+k_{a} z_{\mathrm{IIA}}^{a}\right|-2 \pi i\left(k_{a} A^{a}+n A^{1}\right) .
\end{aligned}
$$

Here we normalized $\Omega$ such that $X^{1}=1, X^{a}=z^{a}$, and the fact that supersymmetry on the worldvolume of the D2-branes constrains $\left(F / 2 \pi-\hat{B}_{\mathrm{NS}}\right)$ to be zero [36].

Comparing the topological couplings in (4.5), (4.7) and (4.6), (4.8) identifies the remaining pieces of the mirror map

$$
A^{1}=\tau_{1}, \quad A^{a}=-\left(c^{a}-\tau_{1} b^{a}\right) .
$$

We remark that the shift of the axion by an element of $H^{0}(Y, \mathbb{Z})$ (i.e., $\tau_{1} \mapsto$ $\tau_{1}+1$, which is part of the $\operatorname{SL}(2, \mathbb{Z})$ symmetry of the type IIB string on $Y$ ) gets identified with the shift of $C_{3}$ by an element of $H^{3,0}(X, \mathbb{Z})$ so that these integral structures of $Y$ and $X$ match [37].

We end this section by summarizing the mirror map that relates the type-IIA and type-IIB variables

$$
\phi_{\mathrm{IIA}}=\phi_{\mathrm{IIB}}, \quad A^{1}=\tau_{1}, \quad A^{a}=-\left(c^{a}-\tau_{1} b^{a}\right), \quad z_{\mathrm{IIA}}^{a}=z_{\mathrm{IIB}}^{a},
$$


where it is understood that type IIB is compactified on the mirror CY such that the Hodge numbers are interchanged. This is precisely the closed string mirror map found in [31] and in the conifold limit [9].

\section{Membrane instantons from mirror symmetry}

In this section, we finally determine the membrane instanton corrections to the LEEA using the mirror symmetry results reviewed in the previous section. As explained in Section 2, the LEEA is completely determined by a single function $\mathcal{L}$, the superspace Lagrangian density, or equivalently by the tensor potential $\chi$, as defined in (2.5).

\subsection{Resummation of $(p, q)$-string contributions in IIB}

The full $(p, q)$-string corrected tensor potential $\chi^{\mathrm{IIB}}$ for type IIB strings compactified on a generic CY $Y$ was found in $[7]$ as the $\mathrm{SL}(2, \mathbb{Z})$-invariant completion of the string tree-level tensor potential $\chi_{\text {tree }}^{\mathrm{IIB}}$. The latter arises through the c-map by evaluating the general formula (we use that $F_{\Lambda}(z)=$ $\partial F / \partial X^{\Lambda}(z)$ in the coordinates $\left.(3.6)\right)$

$$
\chi=4 r^{0} \tau_{2}^{2} \operatorname{Im}\left(F_{1}(z)+\bar{z}^{a} F_{a}(z)\right)
$$

on the vector prepotential of the T-dual IIA compactification on $Y$ (i.e., the prepotential on the moduli space of complexified Kähler deformations of $Y)^{4}$

$$
F_{\text {Kähler }}=F_{\mathrm{cl}}+F_{\mathrm{ws}-\text { pert }}+F_{\mathrm{ws} \text {-inst }} .
$$

The three terms on the right-hand side of the above equation correspond, respectively, to the classical large volume limit prepotential, the four-loop $\sigma$ model correction, and the contribution from worldsheet instantons. Substitution of (5.2) in (5.1) gives rise to a tensor potential which correspondingly can be split into three parts. Each of them is then separately completed into

\footnotetext{
${ }^{4}$ Note that the scheme-dependent terms in the prepotential (quadratic in $X^{\Lambda}$ with real coefficients) do not contribute to the tensor potential. This is analogous to the Kähler potential in the vector multiplet sector, where such terms drop out as well.
} 
an $\mathrm{SL}(2, \mathbb{Z})$ invariant, resulting in the $(p, q)$-string corrected tensor potential

$$
\chi^{\mathrm{IIB}}=\chi_{\mathrm{cl}}^{\mathrm{IIB}}+\chi_{(-1)}^{\mathrm{IIB}}+\chi_{(1)}^{\mathrm{IIB}},
$$

from which the couplings in the effective Lagrangian can be determined [20]. Normalized as in [9], the above terms read

$$
\begin{aligned}
\chi_{\mathrm{cl}}^{\mathrm{IIB}} & =4 r^{0} \tau_{2}^{2} \frac{1}{3 !} \kappa_{a b c} t^{a} t^{b} t^{c}, \\
\chi_{(-1)}^{\mathrm{IIB}} & =\frac{r^{0} \tau_{2}^{1 / 2}}{2(2 \pi)^{3}} \chi_{E}(Y) \sum_{m, n}^{\prime} \frac{\tau_{2}^{3 / 2}}{|m \tau+n|^{3}}, \\
(5.4) \quad \chi_{(1)}^{\mathrm{IIB}} & =-\frac{r^{0} \tau_{2}^{1 / 2}}{(2 \pi)^{3}} \sum_{k_{a}} n_{k_{a}} \sum_{m, n}^{\prime} \frac{\tau_{2}^{3 / 2}}{|m \tau+n|^{3}}\left(1+2 \pi|m \tau+n| k_{a} t^{a}\right) e^{-S_{m, n}} .
\end{aligned}
$$

The above expressions are written in terms of the fields introduced in Section 3.1. $\chi_{E}(Y)$ and $\kappa_{a b c}$ are the Euler number and classical triple intersection form on $Y$, respectively, and the primes in the sums indicate that the $(m, n)=(0,0)$ term is excluded. Moreover

$$
S_{m, n}=2 \pi k_{a}\left(|m \tau+n| t^{a}-i m c^{a}-i n b^{a}\right),
$$

is the action of a $(p, q)$-string wrapped $\operatorname{gcd}(m, n)$ times around a holomorphic 2-cycle in homology $k_{a}$ with respect to the basis $\gamma_{(2)}^{a}$ introduced in Section 3.

The different terms in (5.4) have then the following interpretation: $\chi_{\mathrm{cl}}$ is the classical contribution arising through the c-map from the large volume limit $F_{\mathrm{cl}}$ of $F_{\text {Kähler }}$ in (5.2). It is by itself modular invariant, reflecting the $\mathrm{SL}(2, \mathbb{R})$ invariance of the classical action. $\chi_{(-1)}$, the $\operatorname{SL}(2, \mathbb{Z})$ completion of the perturbative $\sigma$-model correction, encodes the corrections to the couplings in the LEEA arising from $(p, q)$-string maps in which the $(p, q)$-string worldvolume is taken to a point in the CY. It includes the one-loop string corrections, together with an infinite series of $\mathrm{D}(-1)$ instanton corrections. Finally $\chi_{(1)}$, the $\mathrm{SL}(2, \mathbb{Z})$ completion of the worldsheet instanton contributions, encompasses the instantons of the $(p, q)$-string for which the latter's worldvolume wraps holomorphically embedded two-cycles $k_{a} \gamma_{(2)}^{a}$ in the CY (note that instantons of the (1,0)-string are the worldsheet instantons). The multiplicity of these cycles in each homology class is characterized by the Gopakumar-Vafa integers $n_{k_{a}}$.

In order to map the above expressions to the mirror type-IIA compactification, we first perform a Poisson resummation to go to an equivalent representation, which has the virtue of making the role of $\mathrm{D}(-1)-$ and 
D1-brane instantons (i.e., their standard topological couplings to the RR fields) manifest.

As shown in [7] one can resum $\chi_{(-1)}^{\mathrm{IIB}}$ into

$$
\begin{aligned}
\chi_{(-1)}^{\mathrm{IIB}}= & \frac{\chi_{E}(Y)}{2(2 \pi)^{3}} r^{0} \sqrt{\tau_{2}}\left[2 \zeta(3) \tau_{2}^{3 / 2}+\frac{2 \pi^{2}}{3} \tau_{2}^{-1 / 2}+8 \pi \tau_{2}^{1 / 2}\right. \\
& \left.\times \sum_{m \neq 0, n>0}\left|\frac{m}{n}\right| e^{2 \pi i m n \tau_{1}} K_{1}\left(2 \pi|m n| \tau_{2}\right)\right] .
\end{aligned}
$$

The first term arises from worldsheet perturbation theory, the second from the one-loop string correction, while after expanding the Bessel function at large $\tau_{2}$ (weak coupling) one observes that the sum is the contribution from the $\mathrm{D}(-1)$ instantons to the tensor potential, including the perturbative corrections around the instanton background:

$$
\begin{aligned}
\chi_{\mathrm{D}(-1)}^{\mathrm{IIB}}= & \frac{r^{0} \tau_{2}}{2 \pi^{3 / 2}} \chi_{E}(Y) \sum_{m \neq 0, n>0}\left|\frac{m}{n}\right| \\
& \times\left[\sum_{k=0}^{\infty} \frac{\Gamma(3 / 2+k)}{k ! \Gamma(3 / 2-k)}\left(4 \pi|m n| \tau_{2}\right)^{-k-1 / 2}\right] e^{-2 \pi|m n| \tau_{2}+2 \pi i m n \tau_{1}} .
\end{aligned}
$$

In a similar spirit we split off the worldsheet instanton contribution $(m=0)$ in $\chi_{(1)}$ :

$$
\chi_{(1)}^{\mathrm{IIB}}=\chi_{\mathrm{ws}-\mathrm{inst}}^{\mathrm{IIB}}+\chi_{\mathrm{D} 1 \text {-inst }}^{\mathrm{IIB}}
$$

We again perform a Poisson resummation on the unrestricted integer $n$ in $\chi_{\text {D1-inst }}$ (see Appendix A) and obtain

$$
\begin{aligned}
\chi_{\mathrm{D} 1-\text { inst }}^{\mathrm{IIB}}= & -\frac{r^{0} \tau_{2}}{2 \pi^{2}} \sum_{k_{a}} n_{k_{a}} \sum_{m \neq 0, n \in \mathbb{Z}} \\
& \times \frac{|z+n|}{|m|} K_{1}\left(2 \pi\left|m \tau_{2}\right||z+n|\right) e^{2 \pi i m\left(c-\tau_{1}(b+n)\right)},
\end{aligned}
$$


where we have denoted $z=k_{a} z^{a}, b=k_{a} b^{a}$ and $c=k_{a} c^{a}$. Expanding the Bessel function for large $\tau_{2}$, we obtain

$$
\begin{aligned}
\chi_{\text {D1-inst }}^{\text {IIB }}= & -\frac{r^{0} \tau_{2}}{4 \pi^{2}} \sum_{k_{a}} n_{k_{a}} \sum_{m \neq 0, n \in \mathbb{Z}} \frac{|z+n|^{1 / 2}}{|m|^{3 / 2}} \\
& \times\left[1+\sum_{k=1}^{\infty} \frac{\Gamma(3 / 2+k)}{k ! \Gamma(3 / 2-k)}\left(4 \pi\left|m \tau_{2}\right||z+n|\right)^{-k}\right] \\
& \times \exp \left[-2 \pi\left|m \tau_{2}\right||z+n|+2 \pi i m\left(c-\tau_{1}(b+n)\right)\right] .
\end{aligned}
$$

The argument of the exponential reproduces the worldvolume action expected from (4.6) for D1-instantons (with $n$ units of flux) wrapped $m$ times around $k_{a} \gamma_{(2)}^{a}$.

\subsection{Mirror symmetry and membrane instantons in type IIA}

Using the mirror map discussed in Section 4, the expressions obtained in the previous subsection for type IIB strings compactified on $Y$ can now be mapped to a type-IIA compactification on the mirror CY $X$.

We first consider the string tree-level tensor potential $\chi_{\text {tree }}^{\text {IIB }}$ for type IIB compactified on $Y$. It consists of three parts

$$
\chi_{\mathrm{tree}}^{\mathrm{IIB}}\left(z_{\mathrm{IIB}}^{a}\right)=\chi_{\mathrm{cl}}^{\mathrm{IIB}}+\chi_{\mathrm{ws}-\text { pert }}^{\mathrm{IIB}}+\chi_{\mathrm{ws}-\mathrm{In} s t}^{\mathrm{IIB}}
$$

arising from the three terms in the prepotential $F_{\text {Kähler }}\left(z_{\text {IIB }}^{a}\right)$ given in (5.2). In Section 3 we saw that, under the identification (4.3), this prepotential is equal to the prepotential on the moduli space of complex structure deformations of $X$. We then conclude that (5.11) equals the type-IIA tree-level tensor potential (5.1) as computed from the prepotential $F_{\mathrm{cs}}\left(z_{\text {IIA }}^{a}\right)$ on the moduli space of complex structures on the mirror CY $X$. Furthermore, the mirror map in Equation (4.3) provides the map between the NS-NS fields in the type IIB and IIA compactifications.

Next, we turn to matching the string one-loop term $\chi_{\text {loop. }}$ In [6] it was shown that this correction comes with opposite signs in IIA and IIB compactifications on the same CY (consistent with the fact that the tensor structures involved in the dimensional reduction of the ten-dimensional $R^{4}$ terms come with different signs in the type-IIA and -IIB theories [39]). Taking into account that $\chi_{E}(Y)=-\chi_{E}(X)$, we conclude that this term is left 
invariant under mirror symmetry

$$
\chi_{\text {loop }}^{\mathrm{IIA}}=-\frac{1}{24 \pi} r^{0} \chi_{E}(X)=\frac{1}{24 \pi} r^{0} \chi_{E}(Y)=\chi_{\text {loop }}^{\mathrm{IIB}} .
$$

Finally, using the discussion in Section 4, the non-perturbative contributions $\chi_{\mathrm{D}(-1)}^{\mathrm{IIB}}$ and $\chi_{\mathrm{D} 1}^{\mathrm{IIB}}$ can also easily be mapped to the type-IIA compactification.

The $\mathrm{D}(-1)$ instantons, whose contribution is encoded in $\chi_{\mathrm{D}(-1)}^{\mathrm{IIB}}$ are mapped to D2-brane instantons wrapping the cycle $\gamma_{(3)}^{1}$ with associated tensor potential

$$
\chi_{\mathrm{D} 2 / \gamma_{(3)}^{1}}^{\mathrm{IIA}}=-\frac{r^{0} \tau_{2}}{2 \pi^{2}}\left(\frac{\chi_{E}(X)}{2}\right) \sum_{m, n \neq 0}\left|\frac{n}{m}\right| e^{-2 \pi i m n A^{1}} K_{1}\left(2 \pi|m n| \tau_{2}\right),
$$

which follows directly after substitution of the mirror map (4.10) in $\chi_{\mathrm{D}(-1)}^{\mathrm{IIB}}$.

Similarly, D1 instantons wrapping holomorphic cycles in homology $k_{a} \gamma_{(2)}^{a}$ map to D2-brane instantons wrapping special Lagrangian cycles in homology $k_{a} \gamma_{(3)}^{a}+n \gamma_{(3)}^{1}$, whose contribution to the tensor potential is again found by using the mirror map in $\chi_{\mathrm{D} 1}^{\mathrm{IIB}}$ given by (5.9). This results in

$$
\begin{aligned}
\chi_{\mathrm{D} 2 / \gamma_{(3)}^{a}+n \gamma_{(3)}^{1}}^{\mathrm{IIA}}= & -\frac{r^{0} \tau_{2}}{2 \pi^{2}} \sum_{k_{a}} n_{k_{a}} \sum_{m \neq 0, n \in \mathbb{Z}} \frac{|z+n|}{|m|} \\
& \times K_{1}\left(2 \pi\left|m \tau_{2}\right||z+n|\right) e^{-2 \pi i m k_{a} A^{a}} e^{-2 \pi i m n A^{1}},
\end{aligned}
$$

where $z=k_{a} z^{a}$ as above.

We can now combine the potentials (5.13) and (5.14) by introducing vectors

$$
k_{\Lambda}=\left(n, k_{a}\right), \quad z^{\Lambda}=\left(1, z^{a}\right), \quad A^{\Lambda}=\left(A^{1}, A^{a}\right),
$$

$\Lambda \in\{1, a\}$, and write the full non-perturbative IIA result as a sum over vectors $k_{\Lambda}$ weighted by instanton numbers $n_{k_{\Lambda}}$ as follows:

$$
\chi_{\mathrm{A}-\mathrm{D} 2}^{\mathrm{IIA}}=-\frac{r^{0} \tau_{2}}{2 \pi^{2}} \sum_{k_{\Lambda}} n_{k_{\Lambda}} \sum_{m \neq 0} \frac{\left|k_{\Lambda} z^{\Lambda}\right|}{|m|} K_{1}\left(2 \pi \tau_{2}\left|m k_{\Lambda} z^{\Lambda}\right|\right) e^{-2 \pi i m k_{\Lambda} A^{\Lambda}}
$$


Here, the sum over $k_{a}$ now includes the zero-vector $k_{a}=0$, but $k_{\Lambda}=0$ is excluded. The type-IIA instanton numbers read

$$
n_{\left(n, k_{a}=0\right)}=\frac{1}{2} \chi_{E}(X), \quad n_{\left(n, k_{a}\right)}=n_{k_{a}} \text { as in type IIB. }
$$

This formula captures all type-IIA membrane instanton contributions arising from Euclidean D2-branes wrapping the $A$-cycles of the CY.

Collecting our results, we write our final expression for the complete tensor potential encoding the $A$-cycle membrane instanton corrected LEEA for type-IIA compactifications on a generic CY $X$ as

$$
\begin{aligned}
\chi^{\mathrm{IIA}}= & \chi_{\text {tree }}^{\mathrm{IIA}}+\chi_{\text {loop }}^{\mathrm{IIA}}+\chi_{\mathrm{A}-\mathrm{D} 2}^{\mathrm{IIA}} \\
= & 4 r^{0} \tau_{2}^{2} \operatorname{Im}\left[F_{1}\left(z_{\mathrm{IIA}}\right)+\bar{z}_{\mathrm{IIA}}^{a} F_{a}\left(z_{\mathrm{IIA}}\right)\right]-\frac{1}{24 \pi} r^{0} \chi_{E}(X) \\
& -\frac{r^{0} \tau_{2}}{2 \pi^{2}} \sum_{k_{\Lambda}} n_{k_{\Lambda}} \sum_{m \neq 0} \frac{\left|k_{\Lambda} z^{\Lambda}\right|}{|m|} K_{1}\left(2 \pi \tau_{2}\left|m k_{\Lambda} z^{\Lambda}\right|\right) e^{-2 \pi i m k_{\Lambda} A^{\Lambda} .}
\end{aligned}
$$

The latter formula implies that D2-brane instanton corrections to the LEEA for type-IIA compactified on a CY $X$ are determined by two kinds of topological invariants: the Euler characteristic $\chi_{E}(X)$ of the CY $X$, and the Gopakumar-Vafa invariants $n_{k_{a}}$ of the mirror CY $Y$.

\subsection{Superspace description of the instanton corrections}

As already discussed in Section 2, the hypermultiplet sector arising form a type II string compactification on a generic CY can be encoded by the tensor potential $\chi$ or the superspace density $\mathcal{L}$. When implementing symmetries like the $\mathrm{SL}(2, \mathbb{Z})$ invariance of the type IIB string, it is natural to work with $\chi$ since a symmetry of the effective action directly translates into an invariance of $\chi$. When trying to generalize the results above along the lines of the conjecture made in [30] to include also NS5-brane instantons, or motivated by making contact with the hybrid formalism on the heterotic string side, it is desireable to also have a description of the instanton corrections in terms of the projective superspace density $\mathcal{L}$. Since the corresponding derivation is somewhat technical and not very illuminating, we restrict ourselves to giving the final result, while the details are collected in Appendix B.

Defining the vector

$$
\tilde{\eta}^{\Lambda}(\zeta)=\left(n \eta^{0}+m \eta^{1}, \eta^{a}\right),
$$


Equations (B.5) and (B.10) can conveniently be combined into

$$
\begin{aligned}
\mathcal{L}= & \operatorname{Im}\left[\frac{i}{4 \pi^{3}} \sum_{k_{\Lambda}} n_{k_{\Lambda}} \sum_{m>0, n \in \mathbb{Z}} \oint_{\mathcal{C}_{m, n}}\right. \\
& \left.\times \frac{d \zeta}{2 \pi i \zeta} \frac{1}{m^{2}} \frac{\left(\eta^{0}\right)^{2}}{n \eta^{0}+m \eta^{1}} e^{-2 \pi i m k_{\Lambda} \tilde{\eta}^{\Lambda} / \eta^{0}}\right] .
\end{aligned}
$$

Here, the contours $\mathcal{C}_{m, n}$ enclose a zero $\hat{\zeta}_{+}$of $\zeta\left(n \eta^{0}+m \eta^{1}\right)$, and $n_{k_{\Lambda}}$ is given in (5.17). Evaluating the contour integral using Cauchy's Integral formula and computing $\chi$ via $(2.5),(5.20)$ reproduces $\chi_{\mathrm{A}-\mathrm{D} 2}^{\mathrm{IIA}}$ given in $(5.16)$. Note that since both $\mathrm{D}(-1)$ and $\mathrm{D} 1$ instanton corrections in IIB and $A$-type membrane corrections are encoded in the same superspace Lagrangian, our results provide the superspace description for both types of corrections simultaneously.

\section{Discussion and conclusions}

In this paper, we have used mirror symmetry to determine and sum up a class of membrane instanton corrections, drawing on previous results on $\mathrm{D}(-1)$ and D1 instanton corrections for type IIB strings compactified on a generic Calabi-Yau threefold [7]. These non-perturbative corrections encompass the instanton corrections of Euclidean D2-branes wrapping three-cycles ( $A$-cycles) dual to the supersymmetric zero- and two-cycles of the mirror Calabi-Yau and can conveniently be encoded either in terms of the tensor potenial (5.16) or the projective superspace density (5.20). The resulting formulas are completely fixed by the Euler number and the genus zero Gopakumar-Vafa invariants of the mirror Calabi-Yau. On the type IIA side, the instanton corrections thereby contain contributions from both rigid $\left(b_{1}=0\right)$ and non-rigid $\left(b_{1} \neq 0\right)$ three-cycles, since, according to the SYZ interpretation of mirror symmetry [38], the cycle mirror to the zero-cycle has first Betti number $b_{1}=3$. Thus, the mirror result automatically comprises the integrations over the instanton moduli space including an appropriate measure.

The results obtained in this paper rely on the dual corrections on the type IIB side and non-perturbative mirror symmetry. It would be interesting to rederive these corrections from a microscopic IIA computation. This will require an interpretation of the Gopakumar-Vafa invariants of the mirror Calabi-Yau in terms of properties of special Lagrangian three-cycles. A proposal for a topological quantity capturing these properties has recently been 
made by Joyce [40], and it would be interesting to establish a connection between this proposal and mirror symmetry results.

A natural question is how to generalize our results so as to include the remaining membrane instanton corrections arising from the Euclidean D2-branes wrapping supersymmetric $B$-cycles and possibly supersymmetric combinations involving both $A$ - and $B$-cycles. Figure 1 suggests that their form can be deduced from imposing electric-magnetic duality invariance, i.e., invariance under discrete basis transformations in $H_{3}(X, \mathbb{Z})$, on the LEEA. From the structure of (5.16) it is clear that, besides the Gopakumar-Vafa invariants and the Euler number, the full result will also involve additional topological invariants of the Calabi-Yau which encode a suitable multiplicity of the $B$-cycles (or their dual four- and six-cycles on the mirror CalabiYau). Furthermore, implementing these corrections in the low-energy effective action will require a generalization of the tensor multiplet framework employed in this paper, since the corresponding corrections will break (some of) the remaining shift symmetries which are manifest when working with tensor multiplets. Thus, finding the complete membrane instanton corrected low-energy effective action will require progress in formulating offshell $N=2$ supergravity actions as well as a better understanding of the topological invariants on the compactification manifolds. We hope to return to these questions in the future.

\section{Acknowledgments}

We thank Martin Roček for collaboration during the initial stages of this project at the fourth Simons Workshop in Mathematics and Physics. Furthermore, we thank Frederik Denef, Thomas Grimm, Jean Dominique Länge and Pierre Vanhove for useful discussions. DRL is supported by the European Union RTN network MRTN-CT-2004-005104. FS is supported by the European Commission Marie Curie Fellowship no. MEIF-CT-2005-023966. S.V. thanks the Galilei Galileo Institute for Theoretical Physics for its hospitality and partial support. Further support is acknowledged from INTAS contract 03-51-6346.

\section{Appendix A. Poisson resummation revisited}

In this appendix, we give the identities required for resumming the D1 instanton corrections in Section 5.1, following closely and extending 
Appendix B of [9]. They are based on the Poisson resummation formula

$$
\begin{aligned}
\sum_{n \in \mathbb{Z}} f(x+n a) & =\frac{1}{a} \sum_{n \in \mathbb{Z}} \tilde{f}\left(\frac{2 \pi n}{a}\right) e^{2 \pi i n x / a} \text { with } \\
\tilde{f}(k) & =\int_{-\infty}^{\infty} d x f(x) e^{-i k x} .
\end{aligned}
$$

In order to establish Equation (5.9), we apply this identity to

$$
\begin{aligned}
\chi_{\mathrm{D} 1-\mathrm{inst}}^{\mathrm{IIB}}= & -\frac{r^{0} \tau_{2}^{2}}{(2 \pi)^{3}} \sum_{k_{a}} n_{k_{a}} \sum_{m \neq 0} \\
& \times\left[\sum_{n \in \mathbb{Z}} \frac{1}{|m \tau+n|^{3}}\left(1+2 \pi|m \tau+n| k_{a} t^{a}\right) e^{-S_{m, n}}\right]
\end{aligned}
$$

with $S_{m, n}$ given in (5.5). This requires performing an inverse Fourier transform of the summand appearing in square brackets. Comparing to the general formula (A.1), we identify

$$
\begin{aligned}
\tilde{f}(2 \pi n)= & \frac{(2 \pi)^{3}}{\left(\alpha^{2}+(2 \pi n+\gamma)^{2}\right)^{3 / 2}}\left(1+\sqrt{\alpha^{2}+(2 \pi n+\gamma)^{2}} t\right) \\
& \times e^{-\sqrt{\alpha^{2}+(2 \pi n+\gamma)^{2}} t} e^{2 \pi i m c},
\end{aligned}
$$

with $a=1, x=k_{a} b^{a}$, and we set $\alpha=2 \pi m \tau_{2}, \gamma=2 \pi m \tau_{1}, t=k_{a} t^{a}, c=k_{a} c^{a}$.

The inverse Fourier transform of this expression can be found making the following observation. In [9], we gave the following formula for Fourier Cosine transformations [41]

$$
\int_{0}^{\infty} d x \frac{1}{\sqrt{x^{2}+\alpha^{2}}} e^{-\beta \sqrt{x^{2}+\alpha^{2}}} \cos (x y)=K_{0}\left(\alpha \sqrt{\beta^{2}+y^{2}}\right) .
$$

Taking a derivative with respect to the parameter $\alpha$ leads to the identity

$$
\begin{gathered}
\int_{0}^{\infty} d x \frac{1+\beta \sqrt{x^{2}+\alpha^{2}}}{(x+\alpha)^{3 / 2}} e^{-\beta \sqrt{x^{2}+\alpha^{2}}} \cos (x y) \\
=\frac{1}{\alpha} \sqrt{\beta^{2}+y^{2}} K_{1}\left(\alpha \sqrt{\beta^{2}+y^{2}}\right) .
\end{gathered}
$$

Comparing the integrand appearing on the LHS to (A.3), we observe that this is precisely the Fourier transform required for resumming the D1 instantons in (A.2). Carrying out the resummation then leads to the result (5.9). 


\section{Appendix B. Formulating the instanton corrections in projective superspace}

This appendix contains the derivation of the contour integral representation of the instanton corrections given in Equation (5.20). Since both $\mathrm{D}(-1)$ and D1 instanton corrections in IIB and $A$-type membrane corrections are encoded in the same superspace Lagrangian, our results provide the superspace description for both types of corrections simultaneously.

The derivation of this contour formulation is complicated by the fact that Equation (2.5) cannot be solved for $\mathcal{L}$ without performing a non-trivial integration. We will then employ the following strategy. Starting from the instanton contributions in (5.4), we use the relation (2.6) to compute $\mathcal{L}_{x^{I} x^{J}}$. Using Mathematica, the resulting expressions can be integrated twice, and one can explicitly check that the resulting function $\mathcal{L}$ satisfies the supersymmetry constraint (2.3). The corresponding contour integral representation is then found by trial and error. We first derive the contour formulation for the $\mathrm{D}(-1)$ instantons before turning to the $\mathrm{D} 1$ instantons in the next subsection.

\section{B.1. The $\mathrm{D}(-1)$ instanton sector}

We start by splitting the $\mathrm{D}(-1)$ instanton contribution $\chi_{(-1)}^{\mathrm{IIB}}$ into the perturbative worldsheet corrections $m=0, n \neq 0$ and the contribution from $\mathrm{D}(-1)$ instantons

$$
\chi=\frac{r^{0}}{2(2 \pi)^{3}} \chi_{E} \sum_{m \neq 0, n \in \mathbb{Z}} \frac{\tau_{2}^{2}}{|m \tau+n|^{3}} .
$$

Substituting into (2.6), we find ${ }^{5}$

$$
\mathcal{L}_{x^{1} x^{1}}=-\frac{\chi_{E}}{(2 \pi)^{3} r^{0}} \sum_{m>0, n \in \mathbb{Z}} \frac{m^{2} \tau_{2}^{2}-2\left(m \tau_{1}+n\right)^{2}}{\left[m^{2} \tau_{2}^{2}+\left(m \tau_{1}+n\right)^{2}\right]^{5 / 2}} .
$$

Note that this result does not contain the perturbative one-loop correction since, by virtue of the expansion (5.6), this does not contribute to $\mathcal{L}_{x^{1} x^{1}}$. We now read $\tau_{1}, \tau_{2}$ as functions of the tensor multiplet scalars $v, \bar{v}, x$ (cf. Equation (3.4)) and integrate $\mathcal{L}_{x^{1} x^{1}}(x, v, \bar{v})$ with respect to $x^{1}$. In principle, this integration could give rise to two nontrivial integration functions

\footnotetext{
${ }^{5}$ Alternatively this result can be derived by computing $\mathcal{L}_{x^{1} x^{1}}$ based on $\chi_{\mathrm{D}(-1)}^{\mathrm{IIB}}$ given in (5.6) and performing a Poisson resummation.
} 
$g_{i}\left(v^{0}, \bar{v}^{0}, v^{1}, \bar{v}^{1}, x^{0}\right)$ multiplied by terms independent and linear in $x^{1}$. These integration functions encode the one-loop correction which arises from the known contour integal expression (2.8) and will be set to zero in the following. The result can then be written in the following, suggestive form

$$
\mathcal{L}=\frac{\chi_{E}(Y)}{2(2 \pi)^{3}} \sum_{m>0, n \in \mathbb{Z}} \frac{1}{m^{2}} \frac{1}{\left|n \vec{r}^{0}+m \vec{r}^{1}\right|}\left(\eta^{0}\left(\hat{\zeta}_{+}\right)^{2}+\eta^{0}\left(\hat{\zeta}_{-}\right)^{2}\right)
$$

Here

$$
\begin{aligned}
\eta^{I}\left(\hat{\zeta}_{ \pm}\right)= & x^{I}-\frac{n x^{0}+m x^{1}}{2}\left[\frac{v^{I}}{n v^{0}+m v^{1}}+\frac{\bar{v}^{I}}{n \bar{v}^{0}+m \bar{v}^{1}}\right] \\
& \mp \frac{\left|n \vec{r}^{0}+m \vec{r}^{1}\right|}{2}\left[\frac{v^{I}}{n v^{0}+m v^{1}}-\frac{\bar{v}^{I}}{n \bar{v}^{0}+m \bar{v}^{1}}\right],
\end{aligned}
$$

which is valid for all tensor multiplets $I=0, \ldots, h_{1,1}(Y)+1$. One can then check by explicit calculation that (B.3) satisfies the supersymmetry constraint $\mathcal{L}_{x^{I} x^{J}}+\mathcal{L}_{v^{I} \bar{v}^{J}}=0$, Equation (2.3). Thus $\mathcal{L}$ must have a representation in terms of a contour integral (2.2).

Indeed one can verify that (B.3) can be obtained from evaluating

$$
\mathcal{L}=\operatorname{Im}\left[\frac{i \chi_{E}}{(2 \pi)^{3}} \sum_{m>0, n \in \mathbb{Z}} \oint_{\mathcal{C}_{m, n}} \frac{d \zeta}{2 \pi i \zeta} \frac{1}{m^{2}} \frac{\left(\eta^{0}\right)^{2}}{n \eta^{0}+m \eta^{1}}\right]
$$

with the contour $\mathcal{C}_{m, n}$ enclosing the zero $\hat{\zeta}_{+}$of $\zeta\left(n \eta^{0}+m \eta^{1}\right)$,

$$
\hat{\zeta}_{ \pm}=\frac{1}{2\left(n \bar{v}^{0}+m \bar{v}^{1}\right)}\left[\left(n x^{0}+m x^{1}\right) \mp\left|n \vec{r}^{0}+m \vec{r}^{1}\right|\right] .
$$

In order to verify this statement explicitly, one uses Cauchy's integral formula. Noting that the denominator appearing in (B.5) may be written as

$$
\zeta\left(n \eta^{0}+m \eta^{1}\right)=-\left(n \bar{v}^{0}+m \bar{v}^{1}\right)\left(\zeta-\hat{\zeta}_{+}\right)\left(\zeta-\hat{\zeta}_{-}\right)
$$

one finds that the integrand has a simple pole at $\zeta=\hat{\zeta}_{+}$. Evaluating (B.7) at this pole gives $\left.\zeta\left(n \eta^{0}+m \eta^{1}\right)\right|_{\zeta=\hat{\zeta}_{+}}=\left|n \vec{r}^{0}+m \vec{r}^{1}\right|$, while $\left.\eta^{I}(\zeta)\right|_{\zeta=\hat{\zeta}_{+}}$precisely gives rise to (B.4). 


\section{B.2. Superspace description of D1 instantons}

Deriving the contour integral representation for the D1 instantons completely parallels the previous computation. We start from the D1 instanton contribution contained in (5.4)

$$
\begin{aligned}
\chi= & -\frac{r^{0} \tau_{2}^{1 / 2}}{(2 \pi)^{3}} \sum_{k_{a}} n_{k_{a}} \sum_{m \neq 0, n \in \mathbb{Z}} \\
& \times \frac{\tau_{2}^{3 / 2}}{|m \tau+n|^{3}}\left(1+2 \pi|m \tau+n| k_{a} t^{a}\right) e^{-S_{m, n}} .
\end{aligned}
$$

Using Equation (2.6) we compute

$$
\mathcal{L}_{x^{a} x^{b}}=\frac{1}{2 \pi r^{0}} \sum_{k_{a}} n_{k_{a}} k_{a} k_{b} \sum_{m \neq 0, n \in \mathbb{Z}} \frac{1}{|m \tau+n|} e^{-S_{m, n}} .
$$

Again taking the Poincaré fields as functions of $v, \bar{v}, x$, we note that the scalar fields $x^{a}$ enter into this expression as linear terms in $S_{m, n}$ only. This allows to integrate $\mathcal{L}_{x^{a} x^{b}}$ with respect to $x^{a}, x^{b}$. In this step, we again dropped the integration functions independent of and linear in $x^{a}$ which encode the one-loop and $\mathrm{D}(-1)$ brane instanton corrections already determined. The resulting expression for $\mathcal{L}$ is rather cumbersome, but can be written in terms of $\eta^{I}\left(\hat{\zeta}_{ \pm}\right)$similar as in (B.3).

Using the techniques of the previous subsection, one can then explicitly check that the resulting function $\mathcal{L}$ has the contour integral description

$$
\begin{aligned}
\mathcal{L}= & \operatorname{Im}\left[\frac{i}{4 \pi^{3}} \sum_{k_{a}} n_{k_{a}} \sum_{m>0, n \in \mathbb{Z}} \oint_{\mathcal{C}_{m, n}}\right. \\
& \left.\times \frac{d \zeta}{2 \pi \mathrm{i} \zeta} \frac{1}{m^{2}} \frac{\left(\eta^{0}\right)^{2}}{\left(n \eta^{0}+m \eta^{1}\right)} e^{-2 \pi i m k_{a} \eta^{a} / \eta^{0}}\right]
\end{aligned}
$$

where the contours $\mathcal{C}_{m, n}$ are again taken around the zero $\hat{\zeta}_{+}$of $\zeta\left(n \eta^{0}+m \eta^{1}\right)$. Evaluating the contour integral via Cauchy's integral formula, it is then straightforward, but tedious, to check that (B.10) correctly reproduces (B.9). 


\section{References}

[1] E. Witten, Non-Perturbative superpotentials in string theory, Nucl. Phys. B 474 (1996), 343, hep-th/9604030.

[2] J.A. Harvey and G.W. Moore, Superpotentials and membrane instantons, hep-th/9907026.

[3] K. Becker, M. Becker and A. Strominger, Five-branes, membranes and nonperturbative string theory, Nucl. Phys. B 456 (1995), 130, hep-th/9507158.

[4] P. Berglund and P. Mayr, Non-perturbative superpotentials in F-theory and string duality, hep-th/0504058.

[5] T.W. Grimm, Non-perturbative corrections and modularity in $N=1$ type IIB compactifications, arXiv:0705.3253 [hep-th].

[6] D. Robles-Llana, F. Saueressig and S. Vandoren, String loop corrected hypermultiplet moduli spaces, JHEP 0603 (2006), 081, hep-th/0602164.

[7] D. Robles-Llana, M. Roček, F. Saueressig, U. Theis and S. Vandoren, Nonperturbative corrections to $4 D$ string theory effective actions from SL(2,Z) duality and supersymmetry, Phys. Rev. Lett. 98 (2007), 211602 , hep-th/0612027.

[8] H. Ooguri, Y. Oz and Z. Yin, D-branes on Calabi-Yau spaces and their mirrors, Nucl. Phys. B 477 (1996), 407, hep-th/9606112.

[9] F. Saueressig and S. Vandoren, Conifold singularities, resumming instantons and nonperturbative mirror symmetry, arXiv:0704.2229 [hep-th].

[10] H. Ooguri and C. Vafa, Summing up D-instantons, Phys. Rev. Lett. 77 (1996), 3296, hep-th/9608079.

[11] M. Davidse, F. Saueressig, U. Theis and S. Vandoren, Membrane instantons and de Sitter vacua, JHEP 0509 (2005), 065, hep-th/0506097.

[12] N. Halmagyi, I.V. Melnikov and S. Sethi, Instantons, hypermultiplets and the heterotic string, arXiv:0704.3308 [hep-th].

[13] J. Bagger and E. Witten, Matter couplings in $N=2$ supergravity, Nucl. Phys. B 222 (1983), 1. 
[14] S.J. Gates, C.M. Hull and M. Roček, Twisted multiplets and new supersymmetric nonlinear sigma models, Nucl. Phys. B 248 (1984), 157.

[15] A. Karlhede, U. Lindstrom and M. Roček, Selfinteracting tensor multiplets in $N=2$ superspace, Phys. Lett. B 147 (1984), 297.

[16] W.D. Linch and B.C. Vallilo, Covariant $N=2$ heterotic string in four dimensions, JHEP 0703 (2007), 082, hep-th/0611105.

[17] J. Käppeli, S. Theisen and P. Vanhove, Hybrid formalism and topological amplitudes, hep-th/0607021.

[18] N. Berkovits and W. Siegel, Superspace effective actions for $4 D$ compactifications of heterotic and type II superstrings, Nucl. Phys. B 462 (1996), 213, hep-th/9510106.

[19] B. de Wit, M. Roček and S. Vandoren, Hypermultiplets, hyperkaehler cones and quaternion-Kaehler geometry, JHEP 0102 (2001), 039, hep-th/0101161.

[20] B. de Wit and F. Saueressig, Off-shell $N=2$ tensor supermultiplets, JHEP 0609 (2006), 062, hep-th/0606148.

[21] N.J. Hitchin, A. Karlhede, U. Lindstrom and M. Roček, Hyperkahler metrics and supersymmetry, Comm. Math. Phys. 108 (1987), 535.

[22] B. de Wit, B. Kleijn and S. Vandoren, Superconformal hypermultiplets, Nucl. Phys. B 568 (2000), 475, hep-th/9909228.

[23] S. Cecotti, S. Ferrara and L. Girardello, Geometry of type II superstrings and the moduli of superconformal field theories, Internat. J. Mod. Phys. A 4 (1989), 2475.

[24] S. Ferrara and S. Sabharwal, Quaternionic manifolds for type II superstring vacua of Calabi-Yau spaces, Nucl. Phys. B 332 (1990), 317.

[25] M. Roček, C. Vafa and S. Vandoren, Hypermultiplets and topological strings, JHEP 0602 (2006), 062, hep-th/0512206.

[26] M. Roček, C. Vafa and S. Vandoren, Quaternion-Kahler spaces, hyperkahler cones, and the c-map, Handbook of pseudo-Riemannian geometry and supersymmetry, IRMA Lectures in Mathematical Physics, math.dg/0603048.

[27] A. Neitzke, B. Pioline and S. Vandoren, Twistors and black holes, JHEP 0704 (2007), 038, hep-th/0701214. 
[28] S. Alexandrov, Quantum covariant c-map, JHEP 0705 (2007), 094, hep-th/0702203.

[29] I. Antoniadis, R. Minasian, S. Theisen and P. Vanhove, String loop corrections to the universal hypermultiplet, Class. Quant. Grav. 20 (2003), 5079, hep-th/0307268.

[30] L. Anguelova, M. Roček and S. Vandoren, Quantum corrections to the universal hypermultiplet and superspace, Phys. Rev. D 70 (2004), 066001, hep-th/0402132.

[31] R. Böhm, H. Günther, C. Herrmann and J. Louis, Compactification of type IIB string theory on Calabi-Yau threefolds, Nucl. Phys. B 569 (2000), 229, hep-th/9908007.

[32] K. Hori, S. Katz, A. Klemm, R. Pandharipande, R. Thomas, C. Vafa, R. Vakil and E. Zaslow, Mirror symmetry, Clay Mathematics Monographs, Vol. 1, AMS, Providence, USA, 2003.

[33] P. Candelas, X.C. de la Ossa, P.S. Green and L. Parkes, A pair of Calabi-Yau manifolds as an exactly soluble superconformal theory, Nucl. Phys. B 359 (1991), 21.

[34] P.S. Aspinwall, B.R. Greene and D.R. Morrison, Calabi-Yau moduli space, mirror manifolds and spacetime topology change in string theory, Nucl. Phys. B 416 (1994), 414, hep-th/9309097.

[35] D.R. Morrison, Mirror symmetry and rational curves on quintic threefolds: A guide for mathematicians, J. Amer. Math. Soc. 6 (1993), 223, alg-geom/9202004.

[36] M. Mariño, R. Minasian, G.W. Moore and A. Strominger, Nonlinear instantons from supersymmetric p-branes, JHEP 0001 (2000), 005, hep-th/9911206.

[37] D.R. Morrison, Mirror symmetry and the type II string, Nucl. Phys. Proc. Suppl. 46 (1996), 146, hep-th/9512016.

[38] A. Strominger, S.T. Yau and E. Zaslow, Mirror symmetry is T-duality, Nucl. Phys. B 479 (1996), 243, hep-th/9606040.

[39] I. Antoniadis, S. Ferrara, R. Minasian and K.S. Narain, $R^{* *} 4$ couplings in $M$ - and type II theories on Calabi-Yau spaces, Nucl. Phys. B 507 (1997), 571, hep-th/9707013. 
[40] D. Joyce, On counting special Lagrangian homology 3-spheres, Contemp. Math. 314 (2002), 125, hep-th/9907013.

[41] A. Erdélyi (ed.), Tables of integral transforms, Vol. 1, McGraw-Hill Book Company, Inc., New York, 1954.

Institute for Theoretical Physics and Spinoza Institute

UTRECHT UNIVERSITY

3508 TD Utrecht, The Netherlands

E-mail address: D.RoblesLlana@phys.uu.nl

E-mail address: F.S.Saueressig@phys.uu.nl

E-mail address: S.Vandoren@phys.uu.nl

Institute For Theoretical Physics

FrIEDRICH-SCHILLER-UNIVERSITY JENA

D-07743 JENA

GERMANY

E-mail address: Ulrich.Theis@uni-jena.de

Received July 18, 2007; Accepted October 19, 2007 